\title{
PHOTOELECTRIC OBSERVATIONS OF LUNAR OCCULTATIONS. XVI
}

\author{
David S. EVANS AND ANDREW McWiLliaM \\ William H. SANDMANN ${ }^{a)}$ \\ Harvey Mudd College, Claremont, California 91711 \\ MARIAN Frueh \\ McDonald Observatory, Fort Davis, Texas 79734 \\ Received 16 June 1986; revised 25 July 1986
}

McDonald Observatory and the University of Texas at Austin, Austin, Texas 78712-1083

\begin{abstract}
A final list of 105 observations is presented including eight cases of certain or possible duplicity and two angular diameters. The results for 46 Leo (SAO 99172) are combined with previous values to yield a diameter, fully darkened, of $4.01 \pm 0.85$ milliarcsec. A detailed discussion of occultation and speckle results for $\sigma$ Sco is given.
\end{abstract}

\section{INTRODUCTION}

Prime donne are notorious for the number of their farewell performances. The principal investigator in this series, which has been supported mainly by National Science Foundation grants since 1968, apologizes for the fact that Paper XV (Evans et al. 1985) did not clear up all the available observations and that we now present a final 105 results, in Table I, in the usual format, all observed at McDonald Observatory 30 in. reflector in two colors, usually blue clear (BC) and RG 610 filters. The letter following $F$ in the notes column indicates which color was used for reduction. The columns DMS and DMB show the maximum magnitude differences which could have been detected against the star and background light, so that large numbers indicate low noise and high probability of companion detection if one existed. DS is the error associated with the limb slope when one was determined. Asterisks indicate notes to Table I and the designation $\mathrm{R}$ indicates a reappearance.

Table II shows results for duplicity ranked by designation: 3 for certain, 2 for probable, and 1 for possible detection. Table II contains some novel elements. In one case a double solution fits well but it is impossible to tell which component disappeared first, so the position angle is ambiguous by $180^{\circ}$. Two other cases depend on a remark by one of us (Andrew McWilliam) which may throw light on a variety of discrepant results which have turned up in the literature in which impossibly large values of angular diameters have been found. McWilliam suggests that these cases are in reality close binaries. The logic of the matter is that the occultation reduction process seeks to interpret a patch of light on the sky divided into strips parallel to the lunar limb and that it is possible to make the wrong interpretation of a close binary as a star with a sensible diameter. The entries noted in Table II have been tentatively reduced as doubles with the position angles ambiguous by $180^{\circ}$. Angular-diameter determinations are given in Table III.

\section{SIGMA SCORPII DISCUSSED}

The star is ADS 10009, for which we have $\sigma$ Sco A: 2.88, $+0.14,0.70$, B1 III, $\beta$ Cep variable; $\sigma$ Sco B: 8.44 , +0.35 , 0.15 and AB: 3.1, 8.7, 20"., 270 (1783); 20".0, $273^{\circ}$ (1959).

\footnotetext{
a) Visiting Scientist from Harvey Mudd College, Claremont, CA.
}

Evidently B, the distant companion, is physically related.

Occultation observations in this paper and by Nather $e t$ al. (1974) show that $\sigma$ Sco A is itself double. The observation of EB 234 (Eitters and Beavers 1977) in PA 288.2 on 1 January 1973 did not show the secondary but the detection limits $\Delta m_{*}=0.2$ and $\Delta m_{0}=1.9$ indicate that the secondary was probably lost in the noise. Our results show magnitude differences of 2.20 at $5214 \AA$ and 2.41 at $6100 \AA$, while Nather et al. found 2.20 in the blue.

Adopting a magnitude difference of 2.20 we find for the primary of $\sigma$ Sco A 3.03, +0.14 , and +0.70 as the photometric parameters.

The star is evidently considerably reddened and from the standard $(U-B),(B-V)$ plot we can estimate $\Delta(B-V)=0.31$ or $(B-V)_{0}=-0.17$ satisfactorily in agreement with the calibration of Allen (1973), who lists for $\beta$ Cep stars of type B1 III, $(B-V)_{0}=-0.2, \mathrm{M}_{v o}-4.5$, $\log M=1.7$. From the above data we can take the value of $V_{0}$ for this star to be 2.01. The listed photometry for $\sigma$ Sco B is less precise and puts the star in a region of the color-color plot where the determination of reddening is less precise. For it the reddening seems somewhat less, which is possible considering the Milky Way region in which the star lies.

The result from the primary gives a parallax of 0.005 arcsec. If the pair are in orbital motion with a mass of $60 M_{\odot}$ and $a=1$ arcsec the period is $365 \mathrm{yr}$. If $a=0.5 \mathrm{arcsec}$, $P=129 \mathrm{yr}$.

In spite of the vagueness of the foregoing parameter estimates, the conclusion is certain that if there is orbital motion its period is long, possibly of the order of one or several centuries.

On 1972.556, Nather et al. made two observations, both dark-limb disappearances with the secondary going first and found for two different observing stations with our observation added (the results of Table IV). The published position angles of Nather et al. have been increased by $180^{\circ}$ in the light of the order of their events.

If the secondary is slow moving we may combine these using the equation $r \cos \left(\theta-\theta_{n}\right)=r_{n}$ to find the true position angle $\theta$ and separation $r$ from the observed values $\theta_{n}$ and vector separations $r_{n}$. We find $r=0.415$ arcsec, $\theta=276^{\circ} .4$ for a mean date 1977.12. The (O-C) residuals in $r_{n}$ for the three observations are, respectively, $+8,-7$, and -1 milliarcsec. 
TABLE I. Occultation observations.

\begin{tabular}{|c|c|c|c|c|c|c|c|c|c|c|c|c|c|c|c|c|c|c|}
\hline RUN & SAO/DM & MAG & SP. & & .T. & DATE & UTC & OBSERVED & $\mathrm{DT}$ & P.A. & W.A. & C.A. & DMS & DMB & SLOPE & DS & NOTES & EVENT \\
\hline $\begin{array}{l}6759 \\
6760 \\
6761 \\
6762 \\
6763\end{array}$ & $\begin{array}{l}77244 \\
77271 \\
77278 \\
77281 \\
77289\end{array}$ & $\begin{array}{l}8.0 \\
8.5 \\
9.1 \\
7.8 \\
8.8\end{array}$ & $\begin{array}{l}\text { K0 } \\
\text { K7 } \\
\text { A2 } \\
\text { A0 } \\
\text { K5 }\end{array}$ & $\begin{array}{l}01 \\
01 \\
01 \\
01 \\
01\end{array}$ & $\begin{array}{l}\text { MAR } \\
\text { MAR } \\
\text { MAR } \\
\text { MAR } \\
\text { MAR }\end{array}$ & $\begin{array}{ll}R & 85 \\
R & 85 \\
R & 85 \\
R & 85 \\
R & 85\end{array}$ & $\begin{array}{rr}3 & 45 \\
4 & 57 \\
5 & 7 \\
5 & 15 \\
5 & 22\end{array}$ & $\begin{array}{ll}15 & 21.702 \\
57 & 12.745 \\
7 & 10.089 \\
5 & 35.804 \\
2 & 58.524\end{array}$ & $\begin{array}{l}1 \\
1 \\
1 \\
0 \\
1\end{array}$ & $\begin{array}{r}100.1 \\
104.8 \\
90.5 \\
117.5 \\
107.1\end{array}$ & $\begin{array}{r}102.0 \\
106.4 \\
92.0 \\
-26.2 \\
108.8\end{array}$ & $\begin{array}{r}-13.8 \\
-14.1 \\
0.5 \\
119.0 \\
-15.6\end{array}$ & $\begin{array}{l}3.8 \\
3.6 \\
2.4 \\
3.8 \\
2.4\end{array}$ & $\begin{array}{l}3.9 \\
3.6 \\
2.5 \\
4.0 \\
2.5\end{array}$ & $\begin{array}{r}0.0 \\
-2.6\end{array}$ & $\begin{array}{l}5.3 \\
4.8\end{array}$ & $\begin{array}{l}F R(B C, R G) \\
F R(B C, R G) \\
F B(B C, R G) \\
F B(B C, R G) \\
F R(B C, R G)\end{array}$ & \\
\hline $\begin{array}{l}6764 \\
6765 \\
6766 \\
6767 \\
6768\end{array}$ & $\begin{array}{r}77298 \\
77310 \\
77301 \\
77316 \\
+250886\end{array}$ & $\begin{array}{r}9.0 \\
6.3 \\
9.1 \\
9.3 \\
10.3\end{array}$ & $\begin{array}{l}\text { A7 } \\
\text { F5 } \\
\text { A } \\
\text { A } \\
\text { B8 }\end{array}$ & $\begin{array}{l}01 \\
01 \\
01 \\
01 \\
01\end{array}$ & $\begin{array}{l}\text { MAR } \\
\text { MAR } \\
\text { MAR } \\
\text { MAR } \\
\text { MAR }\end{array}$ & $\begin{array}{ll}R & 85 \\
R & 85 \\
R & 85 \\
R & 85 \\
R & 85\end{array}$ & $\begin{array}{lr}5 & 36 \\
5 & 51 \\
5 & 56 \\
6 & 3 \\
6 & 29\end{array}$ & $\begin{array}{rr}36 & 21.120 \\
51 & 38.802 \\
56 & 57.633 \\
3 & 2.916 \\
29 & 57.077\end{array}$ & $\begin{array}{l}1 \\
0 \\
2 \\
1 \\
4\end{array}$ & $\begin{array}{l}73.8 \\
90.0 \\
28.0 \\
73.9 \\
54.7\end{array}$ & $\begin{array}{l}75.1 \\
91.4 \\
29.3 \\
75.3 \\
56.0\end{array}$ & $\begin{array}{r}18.2 \\
2.5 \\
64.5 \\
18.8 \\
38.7\end{array}$ & $\begin{array}{l}2.5 \\
4.3 \\
2.8 \\
2.6 \\
1.4\end{array}$ & $\begin{array}{l}2.6 \\
4.4 \\
2.9 \\
2.7 \\
1.5\end{array}$ & -24.8 & 2.5 & $\begin{array}{l}F B(B C, R G) \\
F B(B C, R G) * \\
F B(B C, R G) \\
F B(B C, R G) \\
F B(B C, R G)\end{array}$ & \\
\hline $\begin{array}{l}6769 \\
6770 \\
6771 \\
6772 \\
6773\end{array}$ & $\begin{array}{l}77335 \\
77337 \\
77360 \\
78361 \\
78381\end{array}$ & $\begin{array}{l}9.1 \\
9.0 \\
5.0 \\
8.8 \\
9.1\end{array}$ & $\begin{array}{l}\text { K5 } \\
\text { G0 } \\
\text { B3 } \\
\text { K7 } \\
\text { A7 }\end{array}$ & $\begin{array}{l}01 \\
01 \\
01 \\
02 \\
02\end{array}$ & $\begin{array}{l}\text { MAR } \\
\text { MAR } \\
\text { MAR } \\
\text { MAR } \\
\text { MAR }\end{array}$ & $\begin{array}{ll}R & 85 \\
R & 85 \\
R & 85 \\
R & 85 \\
R & 85\end{array}$ & $\begin{array}{rr}6 & 33 \\
6 & 36 \\
7 & 26 \\
1 & 5 \\
1 & 17\end{array}$ & $\begin{array}{ll}33 & 45.047 \\
36 & 18.097 \\
26 & 56.614 \\
5 & 42.774 \\
7 & 28.312\end{array}$ & $\begin{array}{l}3 \\
2 \\
1 \\
2 \\
1\end{array}$ & $\begin{array}{r}116.6 \\
69.8 \\
90.0 \\
20.1 \\
82.5\end{array}$ & $\begin{array}{r}118.0 \\
71.0 \\
91.0 \\
16.7 \\
79.1\end{array}$ & $\begin{array}{r}-23.2 \\
23.7 \\
3.9 \\
61.5 \\
0.1\end{array}$ & $\begin{array}{l}1.4 \\
2.2 \\
2.9 \\
2.8 \\
2.5\end{array}$ & $\begin{array}{l}1.5 \\
2.6 \\
3.0 \\
2.9 \\
2.8\end{array}$ & & & $\begin{array}{l}F R(B C, R G) \\
F B(B C, R G) * \\
F B(B C, R G) * \\
F R(B C, R G) \\
F B(B C, R G)\end{array}$ & \\
\hline $\begin{array}{l}6774 \\
6775 \\
6776 \\
6777 \\
6778\end{array}$ & 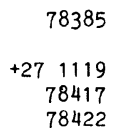 & $\begin{array}{r}9.1 \\
11.2 \\
11.0 \\
6.5 \\
8.8\end{array}$ & $\begin{array}{l}\text { F8 } \\
\text { F0 } \\
\text { K0 } \\
\text { F5 } \\
\text { G5 }\end{array}$ & $\begin{array}{l}02 \\
02 \\
02 \\
02 \\
02\end{array}$ & $\begin{array}{l}\text { MAR } \\
\text { MAR } \\
\text { MAR } \\
\text { MAR } \\
\text { MAR }\end{array}$ & $\begin{array}{ll}R & 85 \\
R & 85 \\
R & 85 \\
R & 85 \\
R & 85\end{array}$ & $\begin{array}{ll}1 & 25 \\
1 & 44 \\
2 & 13 \\
2 & 33 \\
2 & 46\end{array}$ & $\begin{array}{rr}25 & 18.733 \\
+4 & 1.314 \\
13 & 46.434 \\
33 & 42.410 \\
46 & 33.124\end{array}$ & $\begin{array}{l}2 \\
4 \\
4 \\
1 \\
2\end{array}$ & $\begin{array}{r}116.1 \\
63.4 \\
63.7 \\
54.7 \\
42.7\end{array}$ & $\begin{array}{r}112.6 \\
59.9 \\
60.0 \\
51.0 \\
39.0\end{array}$ & $\begin{array}{r}-33.0 \\
20.8 \\
22.7 \\
33.2 \\
46.0\end{array}$ & $\begin{array}{l}2.4 \\
1.5 \\
1.6 \\
4.3 \\
2.5\end{array}$ & $\begin{array}{l}2.5 \\
1.6 \\
1.7 \\
4.4 \\
2.6\end{array}$ & 6.8 & 2.0 & $\begin{array}{l}F B(B C, R G) \\
F B(B C, R G) * \\
F R(B C, R G) \\
F R(B C, R G) * \\
F B(B C, R G)\end{array}$ & \\
\hline $\begin{array}{l}6779 \\
6780 \\
6781 \\
6782 \\
6783\end{array}$ & $\begin{array}{l}78578 \\
80857 \\
80876 \\
80886 \\
80888\end{array}$ & $\begin{array}{l}8.8 \\
8.6 \\
9.0 \\
7.4 \\
9.1\end{array}$ & $\begin{array}{l}\text { A0 } \\
\text { G5 } \\
\text { K0 } \\
\text { K0 } \\
\text { GO }\end{array}$ & $\begin{array}{l}02 \\
05 \\
05 \\
05 \\
05\end{array}$ & $\begin{array}{l}\text { MAR } \\
\text { MAR } \\
\text { MAR } \\
\text { MAR } \\
\text { MAR }\end{array}$ & $\begin{array}{ll}R & 85 \\
R & 85 \\
R & 85 \\
R & 85 \\
R & 85\end{array}$ & $\begin{array}{lr}8 & 0 \\
2 & 36 \\
3 & 33 \\
3 & 56 \\
4 & 0\end{array}$ & $\begin{array}{rr}0 & 15.364 \\
36 & 11.230 \\
33 & 36.979 \\
56 & 8.704 \\
0 & 51.452\end{array}$ & $\begin{array}{l}1 \\
3 \\
3 \\
1 \\
4\end{array}$ & $\begin{array}{l}97.9 \\
96.0 \\
91.9 \\
82.6 \\
82.3\end{array}$ & $\begin{array}{l}93.4 \\
77.8 \\
73.5 \\
64.3 \\
63.9\end{array}$ & $\begin{array}{r}3.0 \\
12.0 \\
19.7 \\
30.5 \\
31.2\end{array}$ & $\begin{array}{l}2.2 \\
1.7 \\
1.8 \\
3.6 \\
1.3\end{array}$ & $\begin{array}{l}2.3 \\
1.8 \\
1.9 \\
3.7 \\
1.4\end{array}$ & 5.1 & 3.1 & $\begin{array}{l}F B(B C, R G) \\
F R(B C, R G) \\
F B(B C, R G) \\
F R(B C, R G) \\
F B(B C, R G)\end{array}$ & \\
\hline $\begin{array}{l}6784 \\
6785 \\
6786 \\
6787 \\
6788\end{array}$ & $\begin{array}{r}80890 \\
80898 \\
98668 \\
+192239 \\
98705\end{array}$ & $\begin{array}{l}8.3 \\
7.6 \\
8.6 \\
9.6 \\
9.3\end{array}$ & $\begin{array}{l}\text { F5 } \\
\text { K0 } \\
\text { G5 } \\
\text { G0 }\end{array}$ & $\begin{array}{l}05 \\
05 \\
05 \\
05 \\
05\end{array}$ & $\begin{array}{l}\text { MAR } \\
\text { MAR } \\
\text { MAR } \\
\text { MAR } \\
\text { MAR }\end{array}$ & $\begin{array}{ll}R & 85 \\
R & 85 \\
R & 85 \\
R & 85 \\
R & 85\end{array}$ & $\begin{array}{ll}4 & 11 \\
4 & 32 \\
7 & 47 \\
8 & 57 \\
9 & 58\end{array}$ & $\begin{array}{rr}11 & 8.441 \\
32 & 19.842 \\
47 & 41.128 \\
57 & 8.934 \\
58 & 26.331\end{array}$ & $\begin{array}{l}1 \\
1 \\
5 \\
1 \\
3\end{array}$ & $\begin{array}{r}64.9 \\
93.1 \\
181.6 \\
140.8 \\
161.6\end{array}$ & $\begin{array}{r}46.5 \\
74.8 \\
163.0 \\
122.1 \\
142.8\end{array}$ & $\begin{array}{r}49.2 \\
22.2 \\
-60.0 \\
-20.1 \\
-42.5\end{array}$ & $\begin{array}{l}3.2 \\
3.0 \\
1.9 \\
2.7 \\
1.4\end{array}$ & $\begin{array}{l}3.3 \\
3.1 \\
2.3 \\
2.8 \\
1.5\end{array}$ & -1.8 & 5.9 & $\begin{array}{l}F B(B C, R G) \\
F R(B C, R G) \\
F R(B C, R G) * \\
F B(B C, R G) \\
F B(B C, R G)\end{array}$ & \\
\hline $\begin{array}{l}6789 \\
6790 \\
6791 \\
6792 \\
6793\end{array}$ & $\begin{array}{l}99144 \\
99159 \\
99172 \\
79142 \\
79921\end{array}$ & $\begin{array}{l}7.5 \\
9.0 \\
5.6 \\
6.8 \\
8.8\end{array}$ & $\begin{array}{l}\text { KO } \\
\text { KO } \\
\text { MO } \\
\text { KO } \\
\text { KO }\end{array}$ & $\begin{array}{l}06 \\
06 \\
06 \\
30 \\
31\end{array}$ & $\begin{array}{l}\text { MAR } \\
\text { MAR } \\
\text { MAR } \\
\text { MAR } \\
\text { MAR }\end{array}$ & $\begin{array}{ll}R & 85 \\
R & 85 \\
R & 85 \\
R & 85 \\
R & 85\end{array}$ & $\begin{array}{ll}3 & 11 \\
3 & 58 \\
5 & 48 \\
5 & 50 \\
2 & 48\end{array}$ & $\begin{array}{rr}14 & 22.660 \\
58 & 16.063 \\
48 & 17.324 \\
50 & 5.608 \\
48 & 8.503\end{array}$ & $\begin{array}{l}1 \\
6 \\
0 \\
1 \\
3\end{array}$ & $\begin{array}{l}121.3 \\
104.7 \\
140.0 \\
112.1 \\
125.3\end{array}$ & $\begin{array}{r}100.3 \\
83.6 \\
118.9 \\
104.5 \\
113.0\end{array}$ & $\begin{array}{r}-6.4 \\
12.8 \\
-16.2 \\
-6.5 \\
-17.7\end{array}$ & $\begin{array}{l}2.7 \\
1.1 \\
4.2 \\
2.9 \\
1.8\end{array}$ & $\begin{array}{l}2.9 \\
1.2 \\
4.3 \\
3.0 \\
1.9\end{array}$ & 1.5 & 8.2 & $\begin{array}{l}\mathrm{FR}(B C, R G)^{*} \\
\mathrm{FB}(B C, R G) \\
\mathrm{FB}(B C, R G)^{*} \\
\mathrm{FR}(B C, R G)^{*} \\
\mathrm{FB}(B C, R G)\end{array}$ & \\
\hline $\begin{array}{l}6794 \\
6795 \\
6796 \\
6797 \\
6798\end{array}$ & $\begin{array}{l}79943 \\
79957 \\
79965 \\
79980 \\
79981\end{array}$ & $\begin{array}{l}9.0 \\
9.1 \\
9.1 \\
8.0 \\
9.1\end{array}$ & $\begin{array}{l}\text { FO } \\
\text { K7 } \\
\text { KO } \\
\text { G5 } \\
\text { A2 }\end{array}$ & $\begin{array}{l}31 \\
31 \\
31 \\
31 \\
31\end{array}$ & $\begin{array}{l}\text { MAR } \\
\text { MAR } \\
\text { MAR } \\
\text { MAR } \\
\text { MAR }\end{array}$ & $\begin{array}{ll}R & 85 \\
R & 85 \\
R & 85 \\
R & 85 \\
R & 85\end{array}$ & $\begin{array}{ll}3 & 44 \\
4 & 46 \\
4 & 5 \\
5 & 27 \\
5 & 41\end{array}$ & $\begin{array}{ll}44 & 35.390 \\
46 & 38.731 \\
59 & 13.426 \\
27 & 22.738 \\
44 & 52.912\end{array}$ & $\begin{array}{l}2 \\
1 \\
1 \\
1 \\
2\end{array}$ & $\begin{array}{r}118.9 \\
149.8 \\
146.1 \\
97.1 \\
145.8\end{array}$ & $\begin{array}{r}106.5 \\
137.5 \\
133.6 \\
84.6 \\
133.3\end{array}$ & $\begin{array}{r}-8.3 \\
-37.5 \\
-33.5 \\
15.6 \\
-33.0\end{array}$ & $\begin{array}{l}2.1 \\
2.7 \\
2.6 \\
3.5 \\
2.3\end{array}$ & $\begin{array}{l}2.2 \\
2.8 \\
2.7 \\
3.6 \\
2.4\end{array}$ & -3.3 & 4.8 & $\begin{array}{l}F B(B C, R G) \\
F R(B C, R G) \\
F B(B C, R G) \\
F R(B C, R G) \\
F B(B C, R G)\end{array}$ & \\
\hline $\begin{array}{l}6799 \\
6800 \\
6801 \\
6802 \\
6803\end{array}$ & $\begin{array}{r}79996 \\
+251867 \\
80011 \\
80597 \\
+22 \quad 2038\end{array}$ & $\begin{array}{r}9.1 \\
11.3 \\
9.1 \\
8.1 \\
11.2\end{array}$ & $\begin{array}{l}\text { G0 } \\
\text { KO } \\
\text { KO } \\
\text { G5 } \\
\text { G5 }\end{array}$ & $\begin{array}{l}31 \\
31 \\
31 \\
01 \\
01\end{array}$ & $\begin{array}{l}\text { MAR } \\
\text { MAR } \\
\text { MAR } \\
\text { APR } \\
\text { APR }\end{array}$ & $\begin{array}{l}85 \\
85 \\
R \\
85 \\
85 \\
R\end{array}$ & $\begin{array}{rr}6 & 6 \\
6 & 10 \\
7 & 3 \\
2 & 8 \\
2 & 17\end{array}$ & $\begin{array}{ll}6 & 12.243 \\
0 & 17.212 \\
3 & 57.787 \\
8 & 46.799 \\
7 & 37.309\end{array}$ & $\begin{array}{l}2 \\
2 \\
2 \\
1 \\
4\end{array}$ & $\begin{array}{r}98.1 \\
103.4 \\
113.1 \\
79.0 \\
144.0\end{array}$ & $\begin{array}{r}85.5 \\
90.6 \\
100.4 \\
62.4 \\
127.5\end{array}$ & $\begin{array}{r}14.6 \\
9.3 \\
-1.2 \\
32.0 \\
-32.4\end{array}$ & $\begin{array}{l}2.1 \\
1.8 \\
1.8 \\
3.4 \\
1.4\end{array}$ & $\begin{array}{l}2.2 \\
1.9 \\
1.9 \\
3.6 \\
1.6\end{array}$ & -5.0 & 2.5 & $\begin{array}{l}F B(B C, R G) \\
F R(B C, R G) \\
F R(B C, R G) \\
F R(B C, R G) \\
F B(B C, R G)\end{array}$ & \\
\hline $\begin{array}{l}6804 \\
6805 \\
6806 \\
6807 \\
6808\end{array}$ & $\begin{array}{r}80622 \\
80645 \\
+21 \quad 1976 \\
80658 \\
80672\end{array}$ & $\begin{array}{r}9.1 \\
8.8 \\
11.1 \\
9.0 \\
9.1\end{array}$ & $\begin{array}{l}\text { K5 } \\
\text { G5 } \\
\text { G5 } \\
\text { F5 } \\
\text { A5 }\end{array}$ & $\begin{array}{l}01 \\
01 \\
01 \\
01 \\
01\end{array}$ & $\begin{array}{l}\text { APR } \\
\text { APR } \\
\text { APR } \\
\text { APR } \\
\text { APR }\end{array}$ & $\begin{array}{ll}R & 85 \\
R & 85 \\
R & 85 \\
R & 85 \\
R & 85\end{array}$ & $\begin{array}{lr}4 & 32 \\
5 & 18 \\
5 & 54 \\
6 & 3 \\
7 & 17\end{array}$ & $\begin{array}{rr}32 & 9.167 \\
8 & 14.858 \\
5 & 17.047 \\
3 & 44.263 \\
7 & 54.297\end{array}$ & $\begin{array}{l}5 \\
1 \\
4 \\
1 \\
1\end{array}$ & $\begin{array}{r}161.5 \\
99.0 \\
120.1 \\
117.8 \\
158.6\end{array}$ & $\begin{array}{r}144.8 \\
82.1 \\
103.3 \\
100.9 \\
141.6\end{array}$ & $\begin{array}{r}-43.2 \\
20.2 \\
-0.8 \\
1.5 \\
-40.5\end{array}$ & $\begin{array}{l}1.2 \\
2.3 \\
1.1 \\
2.9 \\
2.3\end{array}$ & $\begin{array}{l}1.4 \\
2.5 \\
1.2 \\
3.1 \\
2.5\end{array}$ & & & $\begin{array}{l}\mathrm{FB}(B C, R G) * \\
\mathrm{FR}(B C, R G) \\
\mathrm{FB}(B C, R G) \\
\mathrm{FB}(B C, R G) \\
F B(B C, R G) *\end{array}$ & \\
\hline $\begin{array}{l}6809 \\
6810 \\
6811 \\
6812 \\
6813\end{array}$ & $\begin{array}{r}80698 \\
+211994 \\
98894 \\
+182305 \\
98898\end{array}$ & $\begin{array}{r}9.6 \\
9.6 \\
7.9 \\
10.5 \\
9.1\end{array}$ & $\begin{array}{l}\text { K0 } \\
\text { G5 } \\
\text { F5 } \\
\text { G5 }\end{array}$ & $\begin{array}{l}01 \\
01 \\
02 \\
02 \\
02\end{array}$ & $\begin{array}{l}\text { APR } \\
\text { APR } \\
\text { APR } \\
\text { APR } \\
\text { APR }\end{array}$ & $\begin{array}{ll}R & 85 \\
R & 85 \\
R & 85 \\
R & 85 \\
R & 85\end{array}$ & $\begin{array}{ll}8 & 30 \\
8 & 55 \\
2 & 24 \\
2 & 42 \\
2 & 50\end{array}$ & $\begin{array}{ll}30 & 46.823 \\
5 & 38.517 \\
24 & 36.084 \\
2 & 39.610 \\
50 & 25.545\end{array}$ & $\begin{array}{l}2 \\
3 \\
1 \\
6 \\
2\end{array}$ & $\begin{array}{r}96.0 \\
119.0 \\
108.6 \\
137.0 \\
83.6\end{array}$ & $\begin{array}{r}78.9 \\
101.9 \\
88.9 \\
117.1 \\
63.7\end{array}$ & $\begin{array}{r}20.1 \\
-3.7 \\
8.1 \\
-19.0 \\
34.7\end{array}$ & $\begin{array}{l}1.6 \\
1.4 \\
2.6 \\
0.8 \\
2.4\end{array}$ & $\begin{array}{l}1.7 \\
1.5 \\
2.7 \\
0.9 \\
2.5\end{array}$ & & & $\begin{array}{l}F R(B C, R G) \\
F R(B C, R G) \\
F R(B C, R G) \\
F B(B C, R G) \\
F B(B C, R G)\end{array}$ & \\
\hline $\begin{array}{l}6814 \\
6815 \\
6816 \\
6817 \\
6818\end{array}$ & $\begin{array}{r}+182307 \\
98955 \\
98971 \\
98974 \\
98983\end{array}$ & $\begin{array}{l}9.6 \\
3.5 \\
9.3 \\
8.6 \\
8.3\end{array}$ & $\begin{array}{l}\mathrm{G} 5 \\
\mathrm{AOP} \\
\mathrm{G} 5 \\
\mathrm{~K} 2\end{array}$ & $\begin{array}{l}02 \\
02 \\
02 \\
02 \\
02\end{array}$ & $\begin{array}{l}\text { APR } \\
\text { APR } \\
\text { APR } \\
\text { APR } \\
\text { APR }\end{array}$ & $\begin{array}{ll}R & 85 \\
R & 85 \\
R & 85 \\
R & 85 \\
R & 85\end{array}$ & $\begin{array}{ll}3 & 5 \\
6 & 58 \\
8 & 1 \\
8 & 37 \\
8 & 48\end{array}$ & $\begin{array}{rl}5 & 44.094 \\
8 & 41.911 \\
3 & 27.318 \\
77 & 37.561 \\
8 & 27.015\end{array}$ & $\begin{array}{l}5 \\
1 \\
4 \\
1 \\
1\end{array}$ & $\begin{array}{r}115.0 \\
61.0 \\
126.0 \\
155.0 \\
109.8\end{array}$ & $\begin{array}{r}95.1 \\
40.7 \\
105.9 \\
134.8 \\
89.5\end{array}$ & $\begin{array}{r}4.3 \\
63.0 \\
-4.0 \\
-33.9 \\
11.0\end{array}$ & $\begin{array}{l}1.1 \\
4.5 \\
1.0 \\
2.9 \\
2.3\end{array}$ & $\begin{array}{l}1.2 \\
4.6 \\
1.1 \\
3.0 \\
2.5\end{array}$ & 4.5 & 0.5 & $\begin{array}{l}F R(B C, R G) \\
F R(B C, R G) * \\
F B(B C, R G) * \\
F B(B C, R G) * \\
F R(B C, R G) *\end{array}$ & \\
\hline $\begin{array}{l}6819 \\
6820 \\
6821 \\
6822 \\
6823\end{array}$ & $\begin{array}{l}98984 \\
99366 \\
99389 \\
99437 \\
99443\end{array}$ & $\begin{array}{l}7.8 \\
8.8 \\
8.5 \\
8.6 \\
8.3\end{array}$ & $\begin{array}{l}\text { Fo } \\
\text { F8 }\end{array}$ & $\begin{array}{l}02 \\
03 \\
03 \\
03 \\
03\end{array}$ & $\begin{array}{l}\text { APR } \\
\text { APR } \\
\text { APR } \\
\text { APR } \\
\text { APR }\end{array}$ & $\begin{array}{l}R \quad 85 \\
R \quad 85 \\
R \quad 85 \\
R \quad 85 \\
R \quad 85\end{array}$ & $\begin{array}{ll}9 & 21 \\
2 & 26 \\
4 & 1 \\
8 & 47 \\
9 & 1\end{array}$ & $\begin{array}{rr}21 & 7.784 \\
26 & 41.873 \\
4 & 16.064 \\
47 & 4.349 \\
1 & 30.040\end{array}$ & $\begin{array}{l}1 \\
2 \\
1 \\
5 \\
1\end{array}$ & $\begin{array}{r}178.6 \\
91.1 \\
68.0 \\
83.3 \\
135.0\end{array}$ & $\begin{array}{r}158.3 \\
69.4 \\
46.1 \\
61.2 \\
113.0\end{array}$ & $\begin{array}{r}-59.2 \\
29.0 \\
58.0 \\
41.5 \\
-11.1\end{array}$ & $\begin{array}{l}3.5 \\
2.2 \\
3.0 \\
1.2 \\
2.1\end{array}$ & $\begin{array}{l}3.7 \\
2.3 \\
3.1 \\
1.3 \\
2.2\end{array}$ & & & $\begin{array}{l}F B(B C, R G) \\
F B(B C, R G) \\
F B(B C, R G) \\
F R(B C, R G) \\
F B(B C, R G)\end{array}$ & \\
\hline $\begin{array}{l}6824 \\
6825 \\
6827 \\
6828 \\
6830\end{array}$ & $\begin{array}{r}99455 \\
99456 \\
119156 \\
119169 \\
139377\end{array}$ & $\begin{array}{l}7.3 \\
9.0 \\
5.1 \\
7.9 \\
8.1\end{array}$ & $\begin{array}{l}\text { F8 } \\
\text { F8 } \\
\text { A0 } \\
\text { F5 } \\
\text { K5 }\end{array}$ & $\begin{array}{l}03 \\
03 \\
04 \\
04 \\
27\end{array}$ & $\begin{array}{l}\text { APR } \\
\text { APR } \\
\text { APR } \\
\text { APR } \\
\text { JUN }\end{array}$ & $\begin{array}{ll}R & 85 \\
R & 85 \\
R & 85 \\
R & 85 \\
N & 85\end{array}$ & $\begin{array}{ll}9 & 2 \\
9 & 25 \\
8 & 10 \\
8 & 5 \\
4 & 3\end{array}$ & $\begin{array}{ll}23 & 51.497 \\
29 & 57.704 \\
10 & 44.052 \\
1 & 53.515 \\
3 & 23.158\end{array}$ & $\begin{array}{l}1 \\
3 \\
0 \\
1 \\
1\end{array}$ & $\begin{array}{r}64.5 \\
61.2 \\
122.5 \\
83.9 \\
66.9\end{array}$ & $\begin{array}{r}58.7 \\
39.2 \\
100.0 \\
61.5 \\
46.9\end{array}$ & $\begin{array}{r}58.7 \\
61.7 \\
6.1 \\
43.2 \\
59.4\end{array}$ & $\begin{array}{l}3.1 \\
1.9 \\
3.7 \\
2.9 \\
3.8\end{array}$ & $\begin{array}{l}3.3 \\
2.1 \\
3.9 \\
3.1 \\
3.9\end{array}$ & -2.8 & 3.1 & $\begin{array}{l}\mathrm{FR}(B C, R G) * \\
\mathrm{FB}(B C, R G) \\
\mathrm{FB}(52, R G) * \\
\mathrm{FB}(B C, R G) \\
\mathrm{FR}(B C, R G)\end{array}$ & \\
\hline
\end{tabular}


TABLE I. (continued)

\begin{tabular}{|c|c|c|c|c|c|c|c|c|c|c|c|c|c|c|c|c|c|}
\hline RUN & SAO/DM & MAG & SP. & U.T & T. DATE & UTC & OBSERVED & DT & P.A. & W.A. & C.A. & DMS & DMB & SLOPE & DS & NOTES & EVENT \\
\hline $\begin{array}{l}6831 \\
6832 \\
6833 \\
6834 \\
6835\end{array}$ & $\begin{array}{l}139383 \\
139405 \\
139408 \\
139415 \\
158546\end{array}$ & $\begin{array}{l}8.6 \\
7.1 \\
7.6 \\
7.4 \\
7.4\end{array}$ & $\begin{array}{l}\text { G5 } \\
\text { G0 } \\
\text { G5 } \\
\text { F8 } \\
\text { G5 }\end{array}$ & $\begin{array}{l}27 \\
27 \\
27 \\
27 \\
28\end{array}$ & $\begin{array}{ll}\text { JUN } & 85 \\
\text { JUN } & 85 \\
\text { JUN } & 85 \\
\text { JUN } & 85 \\
\text { JUN } & 85\end{array}$ & $\begin{array}{rr}4 & 25 \\
5 & 34 \\
5 & 42 \\
6 & 15 \\
4 & 3\end{array}$ & $\begin{array}{ll}5 & 24.372 \\
4 & 49.955 \\
2 & 54.258 \\
5 & 11.050 \\
3 & 32.147\end{array}$ & $\begin{array}{l}2 \\
0 \\
1 \\
1 \\
1\end{array}$ & $\begin{array}{r}78.3 \\
111.0 \\
102.1 \\
114.4 \\
70.4\end{array}$ & $\begin{array}{l}58.2 \\
91.0 \\
82.3 \\
94.5 \\
53.2\end{array}$ & $\begin{array}{r}46.7 \\
10.0 \\
18.2 \\
4.1 \\
53.7\end{array}$ & $\begin{array}{l}2.6 \\
3.5 \\
3.0 \\
2.9 \\
3.9\end{array}$ & $\begin{array}{l}2.7 \\
3.7 \\
3.1 \\
3.1 \\
4.0\end{array}$ & & & $\begin{array}{l}\mathrm{FB}(\mathrm{BC}, \mathrm{RG}) \\
\mathrm{FB}(\mathrm{BC}, \mathrm{RG}) \\
\mathrm{FB}(\mathrm{BC}, \mathrm{RG}) \\
\mathrm{FB}(\mathrm{BC}, \mathrm{RG})^{*} \\
\mathrm{FB}(\mathrm{BC}, \mathrm{RG})\end{array}$ & \\
\hline $\begin{array}{l}6836 \\
6837 \\
6838 \\
6839 \\
6840\end{array}$ & $\begin{array}{l}159175 \\
159213 \\
159237 \\
184322 \\
184329\end{array}$ & $\begin{array}{l}7.6 \\
8.6 \\
8.1 \\
9.3 \\
4.8\end{array}$ & $\begin{array}{l}\text { F5 } \\
\text { K0 } \\
\text { K2 } \\
\text { F8 } \\
\text { A3 }\end{array}$ & $\begin{array}{l}29 \\
29 \\
29 \\
30 \\
30\end{array}$ & $\begin{array}{ll}\text { JUN } & 85 \\
\text { JUN } & 85 \\
\text { JUN } & 85 \\
\text { JUN } & 85 \\
\text { JUN } & 85\end{array}$ & $\begin{array}{rr}2 & 58 \\
5 & 13 \\
6 & 0 \\
2 & 55 \\
3 & 31\end{array}$ & $\begin{array}{rr}8 & 25.605 \\
3 & 5.375 \\
0 & 25.126 \\
5 & 50.057 \\
1 & 1.980\end{array}$ & $\begin{array}{r}0 \\
1 \\
1 \\
11 \\
1\end{array}$ & $\begin{array}{r}113.4 \\
67.0 \\
76.0 \\
84.1 \\
145.8\end{array}$ & $\begin{array}{r}100.1 \\
54.0 \\
63.0 \\
76.0 \\
137.8\end{array}$ & $\begin{array}{r}9.1 \\
49.2 \\
37.0 \\
32.5 \\
-30.0\end{array}$ & $\begin{array}{l}3.8 \\
2.9 \\
3.1 \\
0.6 \\
3.4\end{array}$ & $\begin{array}{l}3.9 \\
3.1 \\
3.2 \\
0.7 \\
3.5\end{array}$ & -3.3 & 1.3 & $\begin{array}{l}F B(B C, R G) \\
F R(B C, R G) \\
F R(B C, R G) \\
F B(B C, R G) \\
F B(S V, S Y) *\end{array}$ & \\
\hline $\begin{array}{l}6841 \\
6842 \\
6843 \\
6844 \\
6845\end{array}$ & $\begin{array}{l}184345 \\
184347 \\
184958 \\
184993 \\
185001\end{array}$ & $\begin{array}{l}9.0 \\
8.6 \\
9.1 \\
8.5 \\
8.8\end{array}$ & $\begin{array}{l}\text { A0 } \\
\text { A0 } \\
\text { A3 } \\
\text { K2 } \\
\text { B9 }\end{array}$ & $\begin{array}{l}30 \\
30 \\
28 \\
28 \\
28\end{array}$ & $\begin{array}{l}\text { JUN } 85 \\
\text { JUN } 85 \\
\text { JUL } 85 \\
\text { JUL } 85 \\
\text { JUL } 85\end{array}$ & $\begin{array}{ll}4 & 36 \\
4 & 45 \\
3 & 51 \\
4 & 34 \\
4 & 59\end{array}$ & $\begin{array}{ll}6 & 35.769 \\
5 & 56.064 \\
1 & 41.109 \\
4 & 53.827 \\
9 & 19.683\end{array}$ & $\begin{array}{l}7 \\
1 \\
2 \\
1 \\
2\end{array}$ & $\begin{array}{r}149.8 \\
148.1 \\
121.4 \\
71.3 \\
134.8\end{array}$ & $\begin{array}{r}141.8 \\
140.1 \\
117.0 \\
67.0 \\
130.6\end{array}$ & $\begin{array}{r}-36.7 \\
-35.5 \\
-16.6 \\
30.3 \\
-34.9\end{array}$ & $\begin{array}{l}0.9 \\
2.6 \\
2.0 \\
3.0 \\
2.3\end{array}$ & $\begin{array}{l}1.0 \\
2.7 \\
2.1 \\
3.1 \\
2.4\end{array}$ & & & $\begin{array}{l}F B(B C, R G) \\
F B(B C, R G) \\
F B(B C, R G) \\
F R(B C, R G) \\
F B(B C, R G)\end{array}$ & \\
\hline $\begin{array}{l}6846 \\
6847 \\
6848 \\
6849 \\
6850\end{array}$ & $\begin{array}{r}-26 \quad 11875 \\
185020 \\
185033 \\
-26 \quad 11875 \\
185077\end{array}$ & $\begin{array}{l}8.8 \\
7.5 \\
6.1 \\
9.3 \\
8.8\end{array}$ & $\begin{array}{l}\text { K5 } \\
\text { F5 V } \\
\text { A0 } \\
\text { KP } \\
\text { K5 }\end{array}$ & $\begin{array}{l}28 \\
28 \\
28 \\
28 \\
28\end{array}$ & $\begin{array}{l}\text { JUL } 85 \\
\text { JUL } 85 \\
\text { JUL } 85 \\
\text { JUL } 85 \\
\text { JUL } 85\end{array}$ & $\begin{array}{rr}5 & 11 \\
5 & 34 \\
5 & 55 \\
6 & 26 \\
7 & 5\end{array}$ & $\begin{array}{rr}1 & 30.080 \\
4 & 59.772 \\
5 & 41.724 \\
6 & 9.749 \\
5 & 47.925\end{array}$ & $\begin{array}{l}2 \\
0 \\
0 \\
3 \\
1\end{array}$ & $\begin{array}{r}83.0 \\
127.1 \\
107.1 \\
59.7 \\
80.5\end{array}$ & $\begin{array}{r}79.0 \\
123.1 \\
103.1 \\
55.9 \\
76.6\end{array}$ & $\begin{array}{r}15.8 \\
-29.8 \\
-11.1 \\
34.5 \\
11.8\end{array}$ & $\begin{array}{l}2.3 \\
3.7 \\
4.7 \\
1.8 \\
2.7\end{array}$ & $\begin{array}{l}2.4 \\
3.8 \\
4: 8 \\
1.9 \\
2.8\end{array}$ & $\begin{array}{r}5.4 \\
-9.8\end{array}$ & $\begin{array}{l}5.2 \\
1.3\end{array}$ & $\begin{array}{l}F R(B C, R G) \\
F R(B C, R G) * \\
F B(B, R G) * \\
F R(B C, R G) \\
F R(B C, R G)\end{array}$ & \\
\hline $\begin{array}{l}6851 \\
6852 \\
6853 \\
6854 \\
6855\end{array}$ & $\begin{array}{l}186349 \\
186351 \\
186361 \\
187723 \\
187826\end{array}$ & $\begin{array}{l}7.4 \\
8.6 \\
7.5 \\
9.0 \\
9.3\end{array}$ & $\begin{array}{l}\text { KO } \\
\text { KO } \\
\text { K5 } \\
\text { F0 } \\
\text { GO }\end{array}$ & $\begin{array}{l}29 \\
29 \\
29 \\
30 \\
30\end{array}$ & $\begin{array}{l}\text { JUL } 85 \\
\text { JUL } 85 \\
\text { JUL } 85 \\
\text { JUL } 85 \\
\text { JUL } 85\end{array}$ & $\begin{array}{rr}5 & 16 \\
5 & 25 \\
5 & 36 \\
3 & 2 \\
5 & 33\end{array}$ & $\begin{array}{ll}6 & 30.068 \\
5 & 23.758 \\
6 & 47.891 \\
2 & 48.062 \\
3 & 51.500\end{array}$ & $\begin{array}{l}1 \\
3 \\
1 \\
5 \\
7\end{array}$ & $\begin{array}{l}56.7 \\
44.2 \\
42.5 \\
91.3 \\
97.8\end{array}$ & $\begin{array}{r}58.5 \\
46.2 \\
44.5 \\
98.8 \\
105.6\end{array}$ & $\begin{array}{r}34.7 \\
46.4 \\
47.2 \\
1.6 \\
-15.1\end{array}$ & $\begin{array}{l}3.1 \\
2.1 \\
4.0 \\
1.1 \\
1.0\end{array}$ & $\begin{array}{l}3.2 \\
2.3 \\
4.1 \\
1.2 \\
1.1\end{array}$ & 2.5 & 1.5 & $\begin{array}{l}F B(B C, R G) \\
F R(B C, R G) \\
F R(B C, R G) \\
F B(B C, R G) \\
F B(B C, R G)\end{array}$ & \\
\hline $\begin{array}{l}6856 \\
6857 \\
6858 \\
6858 B \\
6859\end{array}$ & $\begin{array}{r}187869 \\
187861 \\
-26 \quad 14840 \\
185755 \\
188613\end{array}$ & $\begin{array}{l}8.8 \\
8.5 \\
8.5 \\
4.4 \\
7.4\end{array}$ & $\begin{array}{l}\text { KO } \\
\text { A0 } \\
\text { A2 } \\
\text { F8 } \\
\text { K0 }\end{array}$ & $\begin{array}{l}30 \\
30 \\
31 \\
25 \\
27\end{array}$ & $\begin{array}{l}\text { JUL } 85 \\
\text { JUL } 85 \\
\text { JUL } 85 \\
\text { AUG } 85 \\
\text { AUG } 85\end{array}$ & $\begin{array}{ll}6 & 46 \\
6 & 49 \\
3 & 54 \\
2 & 54 \\
2 & 31\end{array}$ & $\begin{array}{ll}6 & 21.302 \\
9 & 42.842 \\
4 & 43.835 \\
4 & 15.398 \\
1 & 12.613\end{array}$ & $\begin{array}{l}8 \\
3 \\
9 \\
1 \\
1\end{array}$ & $\begin{array}{l}74.5 \\
36.2 \\
50.2 \\
96.3 \\
73.6\end{array}$ & $\begin{array}{l}82.5 \\
44.4 \\
63.2 \\
96.0 \\
84.9\end{array}$ & $\begin{array}{r}3.2 \\
41.2 \\
32.4 \\
-0.2 \\
9.6\end{array}$ & $\begin{array}{l}0.9 \\
2.6 \\
0.3 \\
3.9 \\
3.2\end{array}$ & $\begin{array}{l}1.0 \\
2.8 \\
0.5 \\
4.0 \\
3.3\end{array}$ & & & $\begin{array}{l}F R(B C, R G) \\
F B(B C, R G) * \\
F B(B C, R G) \\
F R(B C, R G) * \\
F R(B C, R G)\end{array}$ & \\
\hline $\begin{array}{l}6860 \\
6861 \\
6862 \\
6863 \\
6864\end{array}$ & $\begin{array}{l}188722 \\
188778 \\
164601 \\
164697 \\
184336\end{array}$ & $\begin{array}{l}4.8 \\
5.0 \\
6.1 \\
6.0 \\
3.1\end{array}$ & $\begin{array}{l}\text { G5 } \\
\text { G5 } \\
\text { A3 } \\
\text { F2 } \\
\text { B1 }\end{array}$ & $\begin{array}{l}27 \\
27 \\
29 \\
29 \\
30\end{array}$ & $\begin{array}{l}\text { AUG } 85 \\
\text { AUG } 85 \\
\text { AUG } 85 \\
\text { AUG } 85 \\
\text { MAR } 86\end{array}$ & $\begin{array}{ll}6 & 11 \\
7 & 52 \\
3 & 27 \\
9 & 22 \\
9 & 45\end{array}$ & $\begin{array}{ll}1 & 51.152 \\
2 & 59.088 \\
7 & 23.439 \\
2 & 38.008 \\
5 & 23.810\end{array}$ & $\begin{array}{l}0 \\
0 \\
1 \\
0 \\
0\end{array}$ & $\begin{array}{r}52.2 \\
89.0 \\
26.5 \\
70.9 \\
265.5\end{array}$ & $\begin{array}{r}63.9 \\
101.0 \\
45.7 \\
90.4 \\
267.4\end{array}$ & $\begin{array}{r}16.7 \\
-21.7 \\
41.5 \\
-14.8 \\
-150.7\end{array}$ & $\begin{array}{l}4.6 \\
4.4 \\
4.1 \\
4.0 \\
3.5\end{array}$ & $\begin{array}{l}4.7 \\
4.5 \\
4.2 \\
4.1 \\
4.1\end{array}$ & $\begin{array}{l}-6.7 \\
-6.1\end{array}$ & 1.5 & $\begin{array}{l}\mathrm{FR}(B C, R G)^{*} \\
\mathrm{FR}(\mathrm{BC}, \mathrm{RG}) * \\
\mathrm{FB}(\mathrm{BC}, \mathrm{RG}) * \\
\mathrm{FB}(\mathrm{BC}, \mathrm{RG}) \text { * } \\
\mathrm{FB}(52, \mathrm{RG})^{*}\end{array}$ & $\mathbf{R}$ \\
\hline
\end{tabular}

Notes to TABLE I. Italicized numbers are those of other runs on the same star.

Run No. Star

6765 77310 HR 1889, HD 36994, + 25879. 6.49, + 0.43, F5 III.

$6770+77337+25^{\circ} 888$. Double, see Table II

671177360125 Tau, HR 1928, HD 37438, + $25^{\circ} 902$.

$5.18,-0.15,-0.68$, B3 IV. SB 27.864 d. Bright Star Catalogue states occultation triple. Not detected on this run.

6775

6777

6786

6789

6791

6792

6808

6815

6816

6817

6818

6821

6824

6827
USNO No. X09095.

78417 Hamburg (de Vegt 1976) HR 2339, HD 45504, $+27^{\circ} 1122$.

6.47 F5. Double, see Table II

$98668+19^{\circ} 2232$. Double, see Table II.

991446602 .

99172 Poss (1971), 6604, Schmidtke et al. 46 Leo, HR 4127, HD 91232, + $14^{\circ} 2255,5.46$, + 1.68, M 1.5 IIIbCa-I. Diameter, see Table III.

791421313.

80672 Rakos 55 (1971).

$989556612, \eta$ Leo, HR 3975, HD 88737, + 17²171, 3.52, $-0.03,-0.21$ AoIb, ? var?.

989716615 Double, see Table II.

989746617

989836616

993896619

994556621

1191567 Vir, HR 4585, HD 104181, + $4^{\circ} 2556,5.37,-0.00$, $+0.00, \mathrm{~A} 1 \mathrm{~V}$.

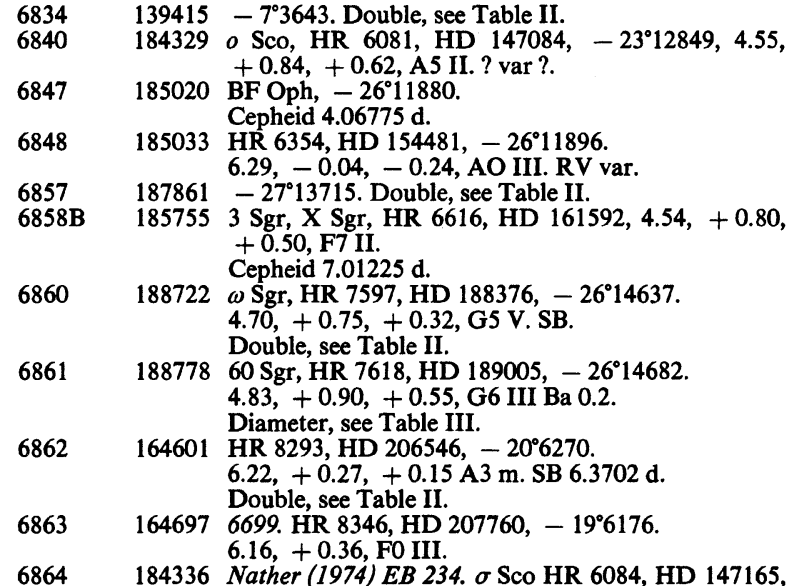

6864184336 Nather (1974) EB 234. $\sigma$ Sco HR 6084, HD 147165, $-25^{\circ} 11485$ ADS
tailed discussion. 
TABLE II. Double star observations.

\begin{tabular}{|c|c|c|c|c|c|c|c|c|c|c|c|}
\hline SAO & Run No. & & T Date & mag & $\mathrm{Sp}$ & $\begin{array}{l}\text { Vector separation } \\
\text { (arc seconds) }\end{array}$ & $\begin{array}{l}\text { P.A. } \\
\text { (deg.) }\end{array}$ & $\Delta \mathrm{m}$ & & Rank & $\begin{array}{l}\text { Slope } \\
\text { (deg.) }\end{array}$ \\
\hline 077337 & 6770 & 01 & Mar 85 & 9.0 & G0 & $\begin{array}{l}0.0271 \pm 0.0016 \\
0.0261 \pm 0.0033\end{array}$ & 249.8 & $\begin{array}{l}0.43 \pm 0.36 \\
0.89 \pm 0.69\end{array}$ & $\begin{array}{l}\text { (BC) } \\
(\mathrm{RG} 610)\end{array}$ & $\begin{array}{l}3 \\
3\end{array}$ & \\
\hline 078417 & 6777 & 02 & Mar 85 & 6.5 & F5 & $\begin{array}{l}0.0040 \pm 0.0005 \\
0.0036 \pm 0.0015\end{array}$ & $\begin{array}{l}61.5^{\mathrm{a}} \\
58.0^{\mathrm{a}}\end{array}$ & $\begin{array}{l}3.40 \pm 1.00 \\
2.40 \pm 0.60\end{array}$ & $\begin{array}{l}\text { (BC) } \\
(\text { RG610) }\end{array}$ & $\begin{array}{l}2 \\
2\end{array}$ & $\begin{array}{l}6.8 \pm 2.0 \\
3.3 \pm 2.0\end{array}$ \\
\hline $\begin{array}{l}098668 \\
139415\end{array}$ & $\begin{array}{l}6786 \\
6834\end{array}$ & $\begin{array}{l}05 \\
27\end{array}$ & $\begin{array}{l}\text { Mar } 85 \\
\text { Jun } 85\end{array}$ & $\begin{array}{l}8.6 \\
7.4\end{array}$ & $\begin{array}{l}\text { G5 } \\
\text { F8 }\end{array}$ & $\begin{array}{l}0.0332 \pm 0.0016 \\
0.7260 \pm 0.0050 \\
0.7252+0.0050\end{array}$ & $\begin{array}{l}181.7 \\
114.4 \\
294.4\end{array}$ & $\begin{array}{l}0.82 \pm 0.40 \\
0.09 \pm 0.19 \\
0.15+0.16\end{array}$ & $\begin{array}{l}\text { (BC) } \\
(\mathrm{BC}) \\
(\mathrm{RG} 610)\end{array}$ & $\begin{array}{l}3 \\
3 \\
3\end{array}$ & \\
\hline $\begin{array}{l}187861 \\
188722\end{array}$ & $\begin{array}{l}6857 \\
6860\end{array}$ & $\begin{array}{l}30 \\
27\end{array}$ & $\begin{array}{l}\text { Jul } 85 \\
\text { Aug } 85\end{array}$ & $\begin{array}{l}8.5 \\
4.8\end{array}$ & $\begin{array}{l}\text { A0 } \\
\text { G5 }\end{array}$ & $\begin{array}{r}0.0968 \pm 0.0015 \\
0.0017 \pm 0.0005 \\
\leqslant 0.0010+0.0005\end{array}$ & $\begin{array}{l}36.3 \\
51.3^{\mathrm{b}} \\
44.9^{\mathrm{b}}\end{array}$ & $0.10 \pm 0.26$ & $\begin{array}{l}\text { (BC) } \\
(\text { BC) } \\
(\text { RG610) }\end{array}$ & $\begin{array}{l}3 \\
1 \\
1\end{array}$ & $\begin{array}{l}-0.9 \pm 2.5 \\
-7.3+1.5\end{array}$ \\
\hline 164601 & 6862 & 29 & Aug 85 & 6.1 & $\mathrm{~A} 3$ & $\begin{array}{l}0.0009 \pm 0.0005 \\
0.0011 \pm 0.0005\end{array}$ & $\begin{array}{l}17.9^{\mathrm{b}} \\
21.6^{\mathrm{b}}\end{array}$ & & $\begin{array}{l}\text { (BC) } \\
(\mathrm{RG} 610)\end{array}$ & $\begin{array}{l}1 \\
1\end{array}$ & $\begin{array}{l}-8.6 \pm 1.5 \\
-4.9 \pm 1.5\end{array}$ \\
\hline 184336 & 6864 & 30 & Mar 86 & 3.1 & B1 & $\begin{array}{l}0.3946 \pm 0.0050 \\
0.3946 \pm 0.0050\end{array}$ & 259.4 & $\begin{array}{l}2.20 \pm 0.07 \\
2.41 \pm 0.16\end{array}$ & $\begin{array}{l}\text { (G5214) } \\
\text { (RG610) }\end{array}$ & $\begin{array}{l}3 \\
3\end{array}$ & $-6.1 \pm 1.0$ \\
\hline
\end{tabular}

a.A. ambiguous to $180^{\circ}$.

${ }^{b}$ Diameter solutions run.

Notes to TABLE II

Star Run No.

0773376770 New double star

0784176777 Hamburg, de Vegt [Astron. Astrophys. 48, 245 (1976) ] has double, separation 3 mas, P.A. $269^{\circ}$ but magnitude difference $0.9 \pm 0.7$

0986686786 New double star
139415

187861 164601

184336
6834 ADS 8950 7.9, 8.4, $01.3127^{\circ}$ (1960) appears to have closed since 1960 .

6857 New double star.

6862 Hyades group. Bright star catalogue has separation 0.001 ", luminosity III.

$6864 \sigma$ Sco, see detailed discussion.
This establishes that the secondary was west of the primary during recent years.

We are indebted to Dr. H. McAlister for making available a list of recent speckle interferometry observations in advance of publication. The standard convention is to state position angles between zero and $180^{\circ}$ and our remarks above show that his published values must be increased by $180^{\circ}$. These modifications have been made in Table V. In our discussion we average the groups in 1980 and 1981 . We also note that the mean occultation position is not in bad agreement with these results.

TABLE III. Angular diameter determinations

\begin{tabular}{|c|c|c|c|c|c|}
\hline Star & Run No. & UT Date & Mag & Sp. & $\begin{array}{c}\text { Angular Diameter }(f d) \\
\text { (milliarcsec) }\end{array}$ \\
\hline 99172 & 6791 & 06 Mar 85 & 5.6 & M0 & $\begin{array}{l}3.9 \pm 1.1(\mathrm{BC}) \\
4.7 \pm 1.0(6100)\end{array}$ \\
\hline 188778 & 6861 & 27 Aug 85 & 5.0 & G5 & $\begin{array}{l}\leqslant 1.2+0.5(\mathrm{BC}) \\
\geqslant 1.2 \pm 0.5(6100)\end{array}$ \\
\hline
\end{tabular}

\section{Notes to TABLE III}

SAO 099172, 46 Leo: Run No. 6604 (noisy): No result.

Other results:

Poss: $5.6 \pm 1.1$ mas (uniform disk) 26.05.66

Schmidtke et al.:

$3.04 \pm 0.76(0.96 \mu)$ (uniform disk)

$3.11 \pm 0.24(1.65 \mu) 19.05 .83$

$3.70 \pm 0.32(2.22 \mu)$

$4.45 \pm 0.62(0.45 \mu)$ (uniform disk)

$3.75 \pm 0.20(0.58 \mu) 12.04 .84$

$2.93 \pm 0.65(0.96 \mu)$

$3.28+0.28(1.65 \mu)$

$2.17 \pm 0.7(2.17 \mu)$

This paper $3.9 \pm 1.1(\mathrm{BC})$

$4.7 \pm 1.0(6100) \quad$ Fully darkened

06.03.85

Adjusting the uniform disk results by $13 \%$ to give fully darkened values, the straight mean for full darkening to the limb is $4.01 \pm 0.85$ mas.
Using the McAlister group observations we find that $r^{-2}$ varies exactly linearly over the range from 1977 to 1984 . Using units of (seconds of arc) $)^{2}$ and rate of change of $\theta$ (degrees per year) we estimate the areal constant $r^{2} \dot{\theta}=0.2610$ with $\dot{\theta}=2.09$ in 1977 and 1.77 in 1984

We can estimate the true values of $r$ and $\theta$ in 1986 by the following iterative procedure. Extrapolate $\dot{\theta}$ to give an average value from 1984 to 1986 to estimate $\theta$. Then require the secondary to be on the 1986 occultation position line and deduce $r$, from which a better value of $\dot{\theta}$ can be inferred. The result for 1986.243 is $r=0.392 \operatorname{arcsec}, \theta=268^{\circ} .5$.

Nather et al. combined their two observations to deduce $r=0.49$ arcsec in P.A. $268^{\circ}$ at 1972.556 . However, this result depends on the concurrence of two position lines inclined to each other at only $9^{\circ}$, admittedly open to criticism. We shall extract better information from their two observations by assuming that they are subject to error and seeking a position for the secondary which best fits them using the iterative procedure. We arrive at $r=0.322$ arcsec, $\theta=296^{\circ} 8$. The residuals in $r$ are +20 and -20 milliarcsec.

There are a number of old visual observations, details of which have kindly been supplied by Mr. J. Churms of the South African Astronomical Observatory.

The most circumstantial of these is by Dawson at La Plata (1931) on 1931.564, who saw a disappearance, primary first ("More than half the light went first") at a calculated vector separation of 0.114 arcsec in P.A. 63.9. Even for a highly skilled visual observer, the estimate of a time interval of about half a second must be accepted with a degree of cau-

TABLE IV. Photoelectric occultation observations.

\begin{tabular}{lll}
\hline \hline Place & P.A. & Vector separation $(\operatorname{arcsec})$ \\
\hline Sutherland & $317^{\circ} .4$ & 0.321 \\
Capetown & $326^{\circ} .1$ & 0.261 \\
McDonald & $259^{\circ} .4$ & 0.395 \\
\hline
\end{tabular}


TABLE V. Speckle observations.

\begin{tabular}{lccl}
\hline \hline Date & $\begin{array}{c}\text { P.A. } \\
(\mathrm{deg})\end{array}$ & $\begin{array}{c}r \\
(\mathrm{arcsec})\end{array}$ & References \\
\hline 1976.471 & 291.8 & 0.326 & Morgan et al. $(1978)$ \\
77.487 & 285.4 & 0.353 & McAlister et al. $(1979)$ \\
& & & \\
80.479 & 277.4 & 0.367 & McAlister et al. $(1983)$ \\
80.482 & 277.9 & 0.367 & McAlister et al. $(1983)$ \\
81.457 & 275.2 & 0.372 & McAlister et al. $(1984)$ \\
81.470 & 277.1 & 0.369 & McAlister et al. $(1984)$ \\
81.473 & 275.6 & 0.369 & McAlister et al. (1984) \\
83.425 & 272.9 & 0.378 & McAlister et al. (unpub.) \\
84.378 & 272.3 & 0.384 & McAlister et al. (unpub.) \\
\hline
\end{tabular}

tion, and the position line based on these data could be shifted in either direction parallel to itself. However, as it happens the particular direction of this position line (see Fig. 1) enables us to get a good estimate of the 1931 position. Assume that the position lies on the northern part of the line. The alternative would involve the orbit having swept round from the southern part and back across the northern part to reach the 1968 position in time and numerically $\dot{\theta}$ would have to be very large. Later discussion will suggest that this is not so. So $\theta$ must be between $64^{\circ}$ and $334^{\circ}$ and $r$ between 0.114 arcsec and infinity. If we guess at a series of values for $\theta, r$ must vary greatly and $\theta$ vary wildly. By trial and error we arrive at a position such that the secondary could have gone from the position line to the speckle assemblage in the time available. The result, necessarily somewhat approximate, is $r=0.4 \operatorname{arcsec}, \theta=350^{\circ}$.

Mr. Churms has analyzed a reappearance on 1860.195 at position angle $314^{\circ}$ with an estimated vector separation of 0.23 arcsec, the components appearing in the order SP. This leads to suggested values near 0.45 in P.A. $20^{\circ}$ naturally with considerable uncertainty.

A reappearance was observed (i.e., "glided") at the Cape on 1968.293 in position angle $244^{\circ}$. Nather et al. say "bright limb reappearance," but as the date was April 16 and the full moon was on April 13 this cannot be so.

An approximate position line has been drawn using the Nather et al. offset of 0.08 arcsec but this could easily be much larger, as any experienced visual observer of occultations will know, being based on an interval estimated as one fifth of a second of time.

There was a bright limb reappearance later in the year at P.A. $285^{\circ}$ but Churms' report does not refer to this event.

A close disappearance was observed at the Union Observatory, Johannesburg, on 1953.630 at P.A. $55^{\circ}$ described as

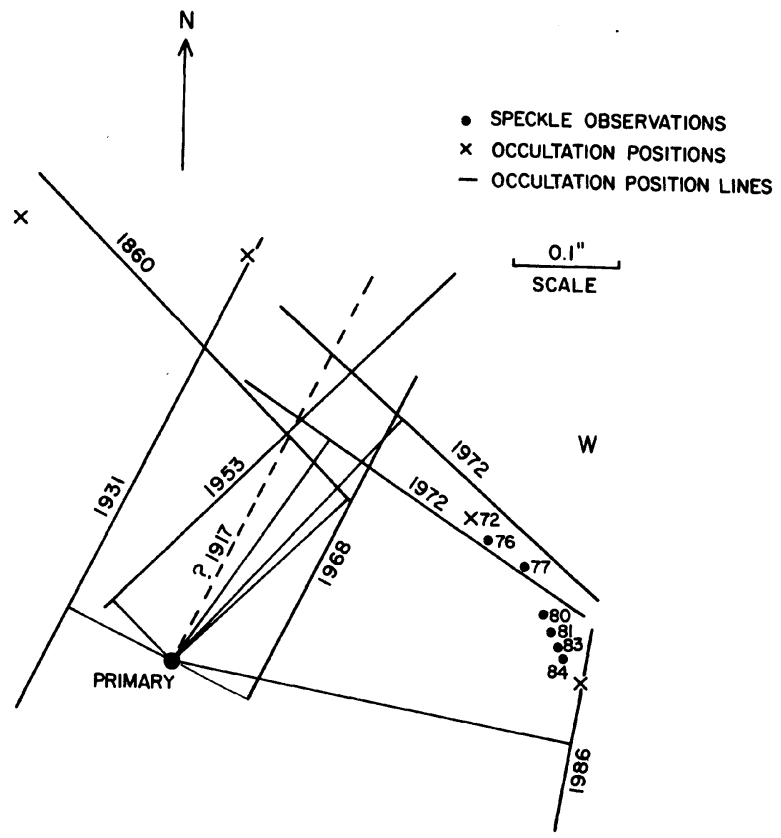

FIG. 1. Positions of the secondary of Sigma Scorpii.

"slow." Again an approximate position line has been drawn. This gets all the events in the right order.

One problem remains. On 1917.274 a dark-limb reappearance was observed at the U.S. Naval Observatory in P.A. $244^{\circ}$ and described as "gradual." We have drawn a position line through the primary in the appropriate direction since we cannot estimate either the amount or direction, east or west, of the vector separation. As it stands, this is inconsistent but it would not take much in the way of alteration of the visual data to get this line to the east of the 1931 one.

The general result gives what seems to be a plausible section of the visual orbit from 1860 to the present time (Fig. 1 ). We will not push matters further at this time, bearing in mind the sage words of the late W. H. van den Bos, who counseled against the deduction of orbits from limited arcs.

It is a pleasure to express our thanks to Dr. H. McAlister and to Mr. J. Churms for information on Sigma Scorpii. This research has been supported by NSF grant No. AST 8309499 and by The University of Texas Research Institute. We are also indebted to Dr. David Dunham and Mrs. M. Lukac for independent computer verification of the occultation circumstances of Sigma Scorpii used in our discussion.

\section{REFERENCES}

Allen, C.W. (1973). Astrophysical Quantities (Athlone, London).

Churms, J. (1986). Private communication.

Dawson, B. (1931). Pop. Astron. 39, 533.

De Vegt, Chr., and Gehlich, U. K. (1976). Astron. Astrophys. 48, 245.

Eitter, J. J., and Beavers, W. I. (1977) Astrophys. J. Suppl. 34, 493.

Evans, D. S., Edwards, D. A., Frueh, M., McWilliam, A., and Sandmann, W. H. (1985). Astron. J. 90, 2360.

McAlister, H. A. (1979). Astrophys. J. 230, 497.

McAlister, H. A., Hendry, E. M., Hartkopf, W. I., Campbell, B. V., and
Fekel, F. C. (1984). Astrophys. J. Suppl. 54, 251.

McAlister, H. A., et al. (1986). Private communication.

Morgan, B. L., Beddoes, D. R., Scaddan, R. J., and Dainty, J. C. (1978). Mon. Not. R. Astron. Soc. 183, 116.

Nather, R. E., Churms, J., and Wild, P. A. T. (1974), Publ. Astron. Soc. Pac. 86, 116.

Poss, H. L. (1971). Highlights Astron. 2, 692.

Schmidtke, P. C., Africano, J. L., Jacoby, G. H., Joyce, R. R., and Ridgway, S. T. (1986). Astron. J. 91, 961. 